\title{
Efeito da Idade sobre a Fertilidade de Éguas Inseminadas com Sêmen Diluído, Resfriado a $14^{\circ} \mathrm{C}$ e Transportado
}

\section{Guilherme Ribeiro Valle ${ }^{2}$, José Monteiro da Silva Filho', Henrique Nunes de Oliveira ${ }^{3}$, Maristela Silveira Palhares ${ }^{1}$, Marcelo Avelar de Mello², Bárbara Goloubeff ${ }^{4}$}

\begin{abstract}
RESUMO - Para estudar o efeito da idade sobre características ovulatórias e de fertilidade, 147 ciclos estrais de 99 éguas foram agrupados em classes por idade (1 - 3-6 anos; 2 - 7-10 anos; 3 - 11-14 anos; e 4 - 15-19 anos), de acordo com o ano do nascimento. As éguas foram inseminadas com sêmen diluído, resfriado e transportado de apenas um garanhão, três vezes por semana (segundas, quartas e sextas-feiras).O controle folicular, por meio da palpação retal, e a rufiação foram realizados diariamente, durante todo o período experimental. Utilizou-se, para o transporte, o sêmen diluído no diluidor de leite desnatado-glicose no conteiner "Celle" modificado, sendo a dose inseminante de $400 \times 10^{6}$ espermatozóides móveis, no momento da diluição final, pré-resfriamento. O tempo médio da coleta do sêmen à inseminação artificial foi de 3,5 horas e a temperatura final do sêmen, no momento da inseminação, de $14^{\circ} \mathrm{C}$. Não houve influência da idade sobre a velocidade de crescimento folicular e o tamanho do folículo ovulatório. A fertilidade decaiu após os 15 anos de idade, traduzida pela diminuição da taxa de concepção/ciclo e eficiência de prenhez, entretanto, não foram observadas características indicativas de senescência até os 19 anos de idade.
\end{abstract}

Palavras-chave: eqüino, idade, fertilidade, inseminação artificial

\section{Effect of Age on Fertility in Mares Inseminated with Diluted, Cooled at $14^{\circ} \mathrm{C}$ and Transported Semen}

\begin{abstract}
To study the effect of age upon ovulatory and fertility traits, 147 cycles of 99 mares were grouped in different age classes (1 - 3-6 years; 2 - 7-10 years; 3 - 11-14 years, and 4 - 15-19 years), according to the year of birth. The mares were inseminated with diluted, cooled and transported semen of one stallion only, three times a week (Monday, Wednesday and Friday). The follicular control, based on rectal palpation, and the teasing were conduced every day, during all the experimental period. For transportation the diluted semen in a skim milk-glucose extender at an insemination dose of $400 \times 10^{6}$ viable spermatozoa was used, before cooling, in the modified "Celle" container. The average semen collection time to the artificial insemination was 3.5 hours and the final temperature of the semen, at the artificial insemination time, of $14^{\circ} \mathrm{C}$. There was no influence of the age on the follicular growth and the size of ovulatory foliculum. The fertility decreases after 15 years of age, with reduction of conception rate/cycle, pregnancy efficiency, however, none characteristics that indicate senescence up to 19 years of age were observed.
\end{abstract}

Key Words: equine, age, fertility, artificial insemination

\section{Introdução}

Os estudos em geriatria eqüina vem sendo intensificados na década de 90. Diferentemente do que ocorre em outras espécies, como a bovina e a suína, as fêmeas eqüinas são mantidas em reprodução por período mais prolongado, embora apenas pequeno percentual do plantel seja representado por esse grupo. GINTHER (1992), compilando dados de quatro países, mostra que as fêmeas acima de 14 anos, mantidas em programas de inseminação artificial, representam 9, 11, 12 e 26\% na Irlanda, nos Estados
Unidos, na Inglaterra e na Austrália, respectivamente. Apesar disso, constituem importante fonte de variação nos índices de fertilidade obtidos, manifestada por alterações ovarianas, tubáricas e uterinas associadas a anormalidades embrionárias (BRINSKO et al., 1995).

A relação negativa entre o avançar da idade e a fertilidade na égua é clara (LOFSTEDT, 1988; VANDERWAL e WOODS, 1991; e CARNEVALE e GINTHER, 1994). No entanto, ainda não está claro se esse efeito é devido ao aumento da ordem de parto ou ao simples envelhecimento. Há correlação positiva entre idade da égua e taxa de perda embrionária, 
que provavelmente obedece à seguinte progressão: 1) reduzida contractilidade e tônus uterino;2) menor habilidade para expulsão de material estranho e bactérias do útero; e 3) maior incidência de endometrites (GINTHER, 1992).

Com relação à atividade cíclica, apesar de muitas éguas ainda ciclarem após 20 anos, há aumento do número de folículos da puberdade até cinco anos, estabilização dos seis aos 15 anos e declínio a partir daí, caminhando para a senescência (GINTHER, 1992). O processo de senescência progride da seguinte forma: a) alongamento da fase folicular e redução do número de folículos; b) ovulações ocasionais (período interovulatório maior que 80 dias); e c) persistência da inatividade ovariana, com folículos menores que 0,5 cm (CARNEVALE e GINTHER, 1994).

O objetivo do presente estudo foi comparar a fertilidade de ciclos estrais de éguas de diferentes faixas etárias, bem como detectar diferenças nas características cíclicas, em função da idade.

\section{Material e Métodos}

O trabalho foi realizado na Zona Metalúrgica do Estado de Minas Gerais, nos municípios de Belo Horizonte e Florestal, distando $60 \mathrm{~km}$ entre si. O período experimental compreendeu duas estações de monta consecutivas (94/95 e 95/96).

Foram utilizados 147 ciclos, de 99 éguas mestiças de 3 a 19 anos de idade, pertencentes a diferentes categorias reprodutivas (éguas solteiras, éguas paridas e potras). Antes do início de cada uma das estações de monta, todas as éguas solteiras e potras foram submetidas a exame ginecológico, constituído de avaliação conformacional externa e interna do sistema genital, citologia e cultura de conteúdo uterino (exceto as potras), e antibiograma, quando o resultado da respectiva cultura o indicava.

As fêmeas foram mantidas no município de Florestal (latitude: $19^{\circ} 02^{\prime}$, longitude: $44^{\circ} 05^{\prime}$ W Gr; temperatura média: $21,8^{\circ} \mathrm{C}$ ). Os controles de crescimento folicular nas éguas foram realizados por palpação retal em dias alternados, até que se detectasse um folículo de 2,0 a 2,5 cm de diâmetro em um dos ovários, sendo, a partir daí, realizados diariamente. Ao se detectar um folículo de 3,0 a 3,5 cm de diâmetro, iniciavam-se as inseminações artificiais, que terminavam quando detectada a ovulação. As éguas e potras obedeciam a esse esquema ao início das estações, porém, as éguas paridas eram palpadas a partir do quinto dia após o parto. Cerca de metade das éguas trabalhadas foi rufiada diariamente a partir da detecção do folículo de 2,0 a 2,5 cm de diâmetro, até o dia da ovulação. $\mathrm{O}$ controle gestacional foi feito por palpações retais aos 17, 21, 25, 30, 35, 40, 45, 60 e 90 dias após a ovulação.

Foi utilizado, nas estações 94/95 e 95/96, um mesmo garanhão da raça Brasileira de Hipismo (7-8 anos de idade) como doador de sêmen, que apresentava bom índice de fertilidade, aferida anteriormente (SILVA FILHO et al., 1993). Em Belo Horizonte, o sêmen foi colhido por intermédio de uma vagina artificial modelo Hannover, às segundas, quartas e sextasfeiras. Após avaliação de motilidade e vigor em microscópio óptico e concentração por contagem em câmara de Newbauer, o sêmen foi diluído, a $37^{\circ} \mathrm{C}$, em diluidor de leite em pó desnatado-glicose (KENNEY et al., 1975). As doses inseminantes foram constituídas por 400 milhões de espermatozóides móveis em $15 \mathrm{~mL}$ de sêmen diluído, as quais foram transportadas em container modelo "Celle" modificado (VALLE, 1997), por, em média, 3,5 horas à temperatura final de $14^{\circ} \mathrm{C}$ para Florestal, onde se encontravam as éguas.

De acordo com a idade das éguas, os resultados de 147 ciclos de 99 éguas inseminadas foram agrupados em: 3-6 anos; 7-10 anos; 11-14 anos; e 15-19 anos.

Com objetivo de se ter maior eficiência na detecção de diferenças entre os grupos experimentais, análises de variância foram utilizadas para os resultados de fertilidade obtidos. Por se tratar de uma variável qualitativa, realizou-se a conversão dos dados em quantitativos, segundo proposição de VOSS et al. (1975), por meio da atribuição de dez pontos à gestação obtida no primeiro ciclo, oito pontos à obtida no segundo ciclo, seis à obtida no terceiro ciclo, quatro à obtida no quarto ciclo, dois à obtida no quinto ciclo e zero às falhas de gestação, resultando em valor numérico médio a cada grupo experimental, chamado eficiência de penhez. A comparação entre as médias foi feita pelo teste Student-Newman-Keuls (SNK). Valores quantitativos foram também submetidos à análise de variância e ao teste SNK. Os resultados relativos a número de IA/ciclo, número de IA/ciclo gestante e número de IA/ciclo vazio, por serem dados descontínuos e de distribuição não normal, foram analisados pelo teste não-paramétrico Kruskal-Wallis. Caso fosse detectada diferença entre os tratamentos, aplicava-se o teste t Student para ordenação das médias. Os dados proporcionais (ciclos/concepção, ciclos/égua gestante, taxa de concepção/ciclo e taxa de concepção total) foram submetidos ao teste Qui-Quadrado para detecção de diferenças entre os tratamentos. 


\section{Resultados e Discussão}

Constata-se, pela análise da Tabela 1, que, após o agrupamento dos ciclos em classes por idade das éguas, não houve diferenças no que se refere às taxas de concepção, ao primeiro ciclo. No entanto, quanto às taxas de concepção/ciclo, o grupo de 15-19 anos mostrou-se inferior aos demais $(\mathrm{p}<0,02)$, Tabela.1.

Parâmetros como tamanho do folículo à ovulação e tempo de crescimento do folículo de $2,75 \mathrm{~cm}$ de diâmetro até a ovulação foram semelhantes entre os grupos, demonstrando ausência de efeito da idade sobre o crescimento folicular e ovulação (Tabela 2).

De acordo com GINTHER (1992), a atividade folicular da égua aumenta da puberdade até os cinco anos estabiliza-se entre 6 e 15 anos e declina, a partir daí, em direção à senescência, que ocorre após 20 anos. A senescência inclui alongamento da fase folicular do ciclo e crescimento mais lento do folículo pré-ovulatório (GINTHER, 1992; CARNEVALE e GINTHER, 1994).

Outros parâmetros de eficiência reprodutiva avaliados (Tabela 2), como número de IA/ciclo, número de IA/ciclo gestante e número de IA/ciclo vazio, foram semelhantes entre os grupos. Por outro lado, o número de ciclos/concepção foi maior $(\mathrm{p}<0,02)$ no grupo de 15-19 anos. A eficiência de prenhez mostrou-se inferior $(\mathrm{p}<0,05)$ no grupo de 15-19 anos em relação aos de 3-6 e 7-10 anos, embora fosse semelhante ao grupo de 11-14 anos (Tabela 2).
Parâmetros de controle (Tabela 2), como intervalo IA/ovulação, volume de sêmen, volume de diluidor, tempo da coleta ao resfriamento e tempo de resfriamento, foram semelhantes entre os grupos. Já a temperatura final do sêmen foi menor $(p<0,05)$ no grupo de 3-6 anos, embora a média desse grupo fosse de apenas $0,7^{\circ} \mathrm{C}$ a menos que a maior dos outros grupos. O tempo de rufiação até IA foi maior $(\mathrm{p}<0,05)$ no grupo de 3-6 anos, em comparação com o grupo de 15-19 anos, com diferença de 11,35 min entre as médias (Tabela 2). Diferenças entre as variáveis de controle, acima descritas, deveram-se exclusivamente ao acaso, já que todos os ciclos foram submetidos às mesmas condições experimentais.

No presente experimento, a partir da avaliação das características cíclicas e ovulatórias das éguas, o declínio na velocidade de crescimento folicular, a partir de 15 anos, não foi detectado. Assim, a senescência parece realmente ocorrer a partir dos 20 anos, conforme GINTHER (1992), pois não foram observadas, nas éguas trabalhadas, características indicativas de sua ocorrência até os 19 anos de idade (Tabela 2).

$\mathrm{O}$ efeito da idade da égua sobre a fertilidade tem sido amplamente demonstrado na literatura, sendo os piores resultados atribuídos aos animais mais velhos (LAING e LEECH, 1975; HENRY, 1981; LOFSTEDT,1988; WAELCHLI, 1990; SILVA, 1991; VANDERWALL e WOODS, 1991; CARNEVALE e GINTHER, 1992; GIBBS e DAVISON, 1992; GINTHER, 1992; CARNEVALE e GINTHER, 1994;

Tabela 1- Efeito da idade sobre a fertilidade, por ciclo estral, de éguas inseminadas com sêmen diluído, resfriado e transportado

Table 1 - Effect of age on fertility, by estral cycle, of inseminated mares with diluted, cooled and transported semen

\begin{tabular}{|c|c|c|c|c|c|c|c|c|}
\hline \multirow{6}{*}{$\begin{array}{l}\text { Ciclo } \\
\text { Cycle }\end{array}$} & \multicolumn{8}{|c|}{$\begin{array}{c}\text { Tratamento } \\
\text { Treatment }\end{array}$} \\
\hline & \multicolumn{2}{|c|}{$\begin{array}{c}3-6 \text { anos } \\
\text { years }\end{array}$} & \multicolumn{2}{|c|}{$\begin{array}{c}7-10 \text { anos } \\
\text { years }\end{array}$} & \multicolumn{2}{|c|}{$\begin{array}{c}\text { 11-14 anos } \\
\text { years }\end{array}$} & \multicolumn{2}{|c|}{$\begin{array}{c}15-19 \text { anos } \\
\text { years }\end{array}$} \\
\hline & № & $\operatorname{Taxa}^{2}$ & № & Taxa & № & Taxa & № & Taxa \\
\hline & ciclos $^{1}$ & concepção & ciclos & concepção & ciclos & concepção & ciclos & concepção \\
\hline & $N$ & Conception & $N$ & Conception & $N$. & Conception & $N$. & Conception \\
\hline & cycles & rate & cycles & rate & cycles & rate & cycles & rate \\
\hline 1 & $21(14)$ & 67,67 & $33(21)$ & 63,64 & $26(14)$ & 53,85 & $19(7)$ & 36,84 \\
\hline 2 & $5(4)$ & 80,00 & $9(5)$ & 55,56 & $10(5)$ & 50,00 & $9(2)$ & 22,22 \\
\hline 3 & $1(0)$ & 0,00 & $2(2)$ & 100,00 & $4(2)$ & 50,00 & $3(1)$ & 33,33 \\
\hline 4 & $1(0)$ & 0,00 & - & - & - & - & $2(0)$ & 0,00 \\
\hline 5 & $1(0)$ & 0,00 & - & - & - & - & $1(0)$ & 0,00 \\
\hline Total & $29(18)$ & $62,07^{\mathrm{a}}$ & $44(28)$ & $63,64^{\mathrm{a}}$ & $40(21)$ & $52,50^{\mathrm{a}}$ & $34(10)$ & $29,41^{b}$ \\
\hline
\end{tabular}

${ }^{1}$ Os números entre parênteses referem-se a éguas gestantes.

${ }_{1}^{1}$ Number between parentesis report to pregnancy mares.

2 Valores em \%.

2 Values in $\%$.

Médias, na linha, seguidas de diferentes letras são diferentes $(P<0,02)$.

Means, within a row, followed by different letters are different $(P<.02)$. 
VALLE et al.

Tabela 2 - Efeito da idade sobre parâmetros reprodutivos de éguas inseminadas com sêmen diluído, resfriado e transportado

Table 2 - Effect of age on reproductive aspects of inseminated mares with diluted, cooling and transported semen Item Tratamento

\begin{tabular}{|c|c|c|c|c|}
\hline & \multicolumn{4}{|c|}{ Treatment } \\
\hline & $\begin{array}{l}\text { 3-6 anos } \\
\text { years }\end{array}$ & $\begin{array}{l}\text { 7-10 anos } \\
\text { years }\end{array}$ & $\begin{array}{l}\text { 11-14 anos } \\
\text { years }\end{array}$ & $\begin{array}{l}\text { 15-19 anos } \\
\text { years }\end{array}$ \\
\hline Número de ciclos & 29 & 44 & 40 & 34 \\
\hline Number of cycles & & & & \\
\hline Idade(anos) & $4,38 \pm 1,15^{\mathrm{a}}$ & $8,71 \pm 0,95^{b}$ & $12,05 \pm 1,18^{\mathrm{c}}$ & $16,82 \pm 1,57^{\mathrm{d}}$ \\
\hline Age (years) & & & & \\
\hline Número IA/ciclo & $2,45 \pm 1,02$ & $2,48 \pm 1,27$ & $2,90 \pm 2,31$ & $2,41 \pm 1,21$ \\
\hline Number of AI/cycle & & & & \\
\hline $\begin{array}{l}\text { Número IA/ciclo gestante } \\
\text { Number AI/pregnancy cycle }\end{array}$ & $2,33 \pm 0,69$ & $2,32 \pm 0,82$ & $2,76 \pm 1,79$ & $2,20 \pm 0,79$ \\
\hline Número IA/ciclo vazio & $2,67 \pm 1,37$ & $2,75 \pm 1,81$ & $3,00 \pm 2,81$ & $2,50 \pm 1,35$ \\
\hline $\begin{array}{l}\text { Number AI/no pregnancy cycle } \\
\mathrm{N}^{\circ} \text { ciclos/concepção }\end{array}$ & $1,61^{\mathrm{a}}$ & $1,57^{\mathrm{a}}$ & $1,91^{\mathrm{a}}$ & $3,4^{b}$ \\
\hline Number of cycle/conception & & & & \\
\hline $\begin{array}{l}\text { Taxa de concepção/ciclo } \\
\text { Conception/cycle rate }\end{array}$ & $0,62^{\mathrm{a}}$ & $0,64^{\mathrm{a}}$ & $0,53^{\mathrm{a}}$ & $0,29^{\mathrm{b}}$ \\
\hline Eficiência de prenhez & $5,93^{\mathrm{a}}$ & $5,96^{\mathrm{a}}$ & $4,80^{\mathrm{ab}}$ & $2,71^{\mathrm{b}}$ \\
\hline Pregnancyefficiency & & & & \\
\hline $\begin{array}{l}\text { Intervalo IA/ovulação(*) } \\
\text { Al/ovulation interval }\end{array}$ & $2,17 \pm 0,93$ & $2,18 \pm 1,08$ & $2,64 \pm 0,90$ & $2,29 \pm 1,12$ \\
\hline $\begin{array}{l}\text { Volume de sêmen }(\mathrm{mL}) \\
\text { Semen volume }\end{array}$ & $3,76 \pm 1,61$ & $3,65 \pm 1,36$ & $4,02 \pm 1,62$ & $3,94 \pm 1,89$ \\
\hline $\begin{array}{l}\text { Volume de diluidor }(\mathrm{mL}) \\
\text { Extender volume }\end{array}$ & $11,26 \pm 1,63$ & $11,33 \pm 1,38$ & $10,99 \pm 1,62$ & $11,19 \pm 1,54$ \\
\hline $\begin{array}{l}\text { Tempo coleta/resfriamento(min) } \\
\text { Collection/cooling time }\end{array}$ & $50,07 \pm 12,08$ & $49,53 \pm 13,95$ & $47,45 \pm 12,09$ & $45,93 \pm 9,43$ \\
\hline $\begin{array}{l}\text { Tempo de resfriamento(min) } \\
\text { Cooling time }\end{array}$ & $232,48 \pm 54,60$ & $228,12 \pm 54,01$ & $219,44 \pm 53,31$ & $228,79 \pm 51,58$ \\
\hline $\begin{array}{l}\text { Temperatura do sêmen }\left({ }^{\circ} \mathrm{C}\right) \\
\text { Semen temperature }\end{array}$ & $13,06 \pm 1,80^{\mathrm{a}}$ & $13,90 \pm 1,92^{\mathrm{b}}$ & $14,03 \pm 2,07^{\mathrm{b}}$ & $13,99 \pm 1,81^{\mathrm{b}}$ \\
\hline $\begin{array}{l}\text { Tempo rufiação/IA(min) } \\
\text { Teasing/AI time (min) }\end{array}$ & $42,18 \pm 19,32^{\mathrm{a}}$ & $36,64 \pm 17,66^{\mathrm{ab}}$ & $35,05 \pm 14,36^{\mathrm{ab}}$ & $30,83 \pm 15,80^{\mathrm{b}}$ \\
\hline $\begin{array}{l}\text { Tempo colheita/IA (min) } \\
\text { Collection/AI time (min) }\end{array}$ & $308,96 \pm 63,07$ & $302,41 \pm 63,23$ & $288,32 \pm 60,10$ & $294,07 \pm 57,74$ \\
\hline $\begin{array}{l}\text { Folículo } 2,75 \text { à ovulação (dias) } \\
2.75 \text { follicle to ovulation (days) }\end{array}$ & $5,43 \pm 2,10$ & $5,81 \pm 3,24$ & $5,78 \pm 4,94$ & $5,13 \pm 2,19$ \\
\hline $\begin{array}{l}\text { * Significado apenas para demonstrar } \\
\text { Médias na linha seguidas de diferer } \\
\text { Médias, na linha, para número de c } \\
\text { Significancy only to demonstrate similarity } \\
\text { Means, within a row, followed by differen }\end{array}$ & $\begin{array}{l}\text { entre grupos } \\
\text { ço diferentes }( \\
\text { ça, seguidas } \\
\text { ps }(1=24 P ; 2=48 \\
\text { ferent }(P<.05) \text {. }\end{array}$ & $\begin{array}{l}4 \mathrm{P} ; 2=48 \mathrm{P} ; 3=4 \\
\text { 55). } \\
\text { liferentes letras } \\
48 P P ; 4=72 P P) .\end{array}$ & $\begin{array}{l}4=72 \mathrm{PP}) . \\
\text { diferentes }(\mathrm{P}<0 \text {, }\end{array}$ & \\
\hline
\end{tabular}

ALLEN, 1994; e BRINSKO et al., 1995) e em animais muito jovens (VLACHOS e PASCHALERI, 1965; LOFSTEDT, 1988).

LOFSTEDT (1988) e GIBBS e DAVISON (1992) afirmam que a fertilidade cai a partir de 15-16 anos; VANDERWALL e WOODS (1991), a partir de 12 anos; e LAING e LEECH (1975), a partir de dez anos.

No presente experimento, observou-se que a fertilidade decaiu a partir dos 15 anos de idade, como demonstrado nas Tabelas 1 e 2. Estes resultados estão de acordo com LOFSTEDT (1988) e GIBBS e DAVISON (1992), no entanto, estão em desacordo com as observações de VLACHOS e PASCHALERI (1965), segundo os quais éguas entre 3 e 5 anos apresentaram fertilidade inferior à observada em éguas de 6 a 8 anos.

Os resultados de VLACHOS e PASCHALERI (1965) são, sem dúvida, dignos de atenção, pois são oriundos de observações de 1204 éguas. No entanto, nesse trabalho, o momento de IA das éguas baseouse na detecção de cio, e não no controle folicular, Esses autores afirmam que as éguas de 6-8 anos apresentaram sinais de cio mais pronunciados. Assim, pode ter sido observada fertilidade inferior 
Rev. bras. zootec.

nas éguas de 3-5 anos em decorrência da pior observação de cio, o que não ocorreu no presente experimento. Neste trabalho, as IAs foram realizadas com base no controle folicular, e não na apresentação de cio.

Observações de LAING e LEECH (1975) e VANDERWALL e WOODS (1991) de que a fertilidade decai a partir de 10-12 anos não foram confirmadas neste trabalho. No entanto, observa-se, na Tabela 2, que a eficiência de prenhez do grupo de 11-14 anos foi intermediária às dos grupos etários imediatamente acima (15-19 anos) e abaixo (7-10 anos). Esse pode ser um indício de que a fertilidade já inicia, mesmo que discretamente, queda a partir de 11-14 anos de idade.

A redução da fertilidade, à medida que avança a idade, tem sido atribuída a diversos fatores. Segundo GINTHER (1992), com a idade, advém menor contratilidade uterina e, assim, menor habilidade para expulsar corpos estranhos do útero e, conseqüentemente, maior ocorrência de endometrites. Hughes e Loy (1969), citados por ALLEN (1994), EVANS et al. (1987) e CARNEVALE e GINTHER (1994) verificaram menor capacidade de éguas mais velhas, ao eliminarem corpos estranhos e bactérias da luz uterina, contribuindo para maior susceptibilidade a infecções e alterações inflamatórias e degenerativas.

SILVA (1991), estudando a ocorrência de diversas patologias genitais em relação à idade da égua, verificou, de maneira geral, maior ocorrência com o avançar da idade. As lesões inflamatórias uterinas foram significativamente relacionadas à menor fertilidade. O mesmo foi observado por HENRY (1981), que também cita as salpingites como mais freqüentes em animais mais velhos.

A maior ocorrência de patologias degenerativas, como degenerações crônicas do endométrio (LEISHMAN etal., 1982; SILVA, 1991; CARNEVALE e GINTHER, 1992; HELD e ROHRBACH, 1992; e CARNEVALE e GINTHER, 1994) e cistos uterinos (GINTHER, 1992; TANNUS e THUN, 1995), também estão relacionados ao envelhecimento da égua.

No presente experimento, procurou-se eliminar a interferência destes diversos fatores, como endometrites, patologias vulvo-vestíbulo-vaginais e cistos uterinos, principalmente, por intermédio dos exames ginecológicos, tratamentos e descartes prévios ao experimento. No entanto, endometrites que porventura se instalassem no decorrer do experimento foram tratadas como no manejo normal de uma estação de monta, sendo consideradas como parte integrante do período experimental.

Portanto, a expressão da subfertilidade das éguas mais velhas, por intermédio de endometrites, foi per- mitida durante o período experimental, sendo possível admitir que esta tenha contribuído para os piores resultados do grupo de maior faixa etária.

Segundo VANDERWALL e WOODS (1991), CARNEVALE E GINTHER (1992), GINTHER (1992) e HELD e ROHRBACH (1992), as diversas patologias uterinas são causadoras da maior taxa de perdas embrionárias em éguas mais velhas. No entanto, CARNEVALE e GINTHER (1994) e BRINSKO et al. (1995) verificaram que ovócitos de éguas mais velhas já estão comprometidos, assim como seus embriões, independentemente da condição uterina.

Esta última observação vem reafirmar as suspeitas de LEISHMAN et al. (1982), WAELCHLI (1990) e GINTHER (1992) de que algum fator inerente ao próprio envelhecimento estaria atuando como redutor da fertilidade na égua velha, além da condição uterina.

No presente experimento, em virtude de terem sido feitos os diagnósticos de gestação por meio de palpação retal e rufiações de retorno, as perdas gestacionais anteriores a 21 dias, fase em que já se dava o diagnóstico definitivo de gestação, não puderam ser detectadas.

Caso tivessem sido feitos diagnósticos mais precoces de gestação, perdas gestacionais mais precoces poderiam ter sido detectadas e, em consequiência, ciclos dados como vazios, neste estudo, teriam sido dados como gestantes, sendo os efeitos da idade da égua relativos à condição uterina eliminados da análise. Assim poder-se-ia, então, detectar os efeitos pré-uterinos da idade sobre a fertilidade das éguas, mas não expressariam condição total da égua velha sobre sua fertilidade, como ocorreu no presente experimento.

\section{Conclusões}

A avaliação das características ovulatórias e dos resultados de fertilidade, neste trabalho, permitiram concluir que a fertilidade das éguas decaiu a partir dos 15 anos de idade; entretanto, a senescência não ocorreu até os 19 anos. Assim, esses parâmetros devem ser considerados quando da avaliação de éguas, quanto à fertilidade, para efeito de reposição de rebanho, como receptora ou doadora de embriões.

\section{Referências Bibliográficas}

ALLEN, W.R. Equine endometritis: an update on recent findings and treatment regimes. EQUINE PRACTITIONERS GROUP CONGRESS, 1994, Vancouver. Separata...Vancouver: American Association of Equine Practitioners, 1994. p.72-83. 
BRINSKO, S.P., BALL, B.A., ELLINGTON, J.E. 1995. In vitro maturation of equine oocytes obtained from different age groups of sexually mature mares. Theriogenology, 44(4):461-469.

CARNEVALE, E.M., GINTHER, O.J. 1992. Relationships of age to uterine function and reproductive efficiency in mares. Theriogenology, 37(5):1101-1115.

CARNEVALE, E.M., GINTHER, O.J. Reproductive function in old mares. In: AMERICAN ASSOCIATION OF EQUINE PRACTITIONERS, ANNUAL CONVENTION, 37, 1991, Vancouver. Proceedings.... Vancouver: American Association of Equine Practitioners, 1994. p.15.

EVANS, M.J., HAMER, J.M., GASON, L.M. et al. 1987. Factors affecting uterine clearence of inoculated materials in mares. J. Reprod. and Fert., 35:327-334 (Supplement).

GIBBS, P.G., DAVISON, K.E. 1992. A field study on reproductive efficiency of mares maintained predominately on native pasture. Eq. Vet. J., 12(4):219-222.

GINTHER, O.J. 1992. Reproductive biology of the mare (basic and applied aspects). 2 ed. Cross Plains: Equiservices, 642p.

HELD, J.P.E., ROHRBACH, B.W. Ability of endometrial biopsy results, parity, and age to predict subsequent pregnancy in the mare. In: ANNUAL CONVENTION OF AMERICAN ASSOCIATION OF EQUINE PRACTITIONERS, 37, 1991, San Francisco. Proceedings.... Lexinton: American Association of Equine Practitioners, 1992. p.183-188.

HENRY, M.R.J-M. Some special aspects of the physiopathology of reproduction in mares. Rijksuniversiteit Gent, 1981. 219p. Thesis (Doctor), 1981.

KENNEY, R.M., BERGMAN, R.V., COOPER, W.L. et al. Minimal contamination techniques breeding mares: technique and preliminary findings. Proceedings of the American Association of Equine Practitioners, v.21, p.327-336, 1975.

LAING, J.A., LEECH, F.B. 1975. The frequency of infertilty in thoroughbred mares. J. Reprod. and Fert., 23:307-310. (Supplement).

LEISHMAN, D., MILlER, R.B., DOIG, P.A. 1982. A quantitative study of the histological morphology of the endometrium of normal and barren mares. Can. J. Comparative Med., 46(1):17-20.

LOFSTEDT, R.M. Breeding programs in the mare. In: LAING, J.A., MORGAN, W.J.B., WAGNER, W.C. (Ed.) Fertility \& infertility in veterinary practice. 4 ed. London: Baillière Tindall, 1988. p.140-159.

SILVA, M.I.F. Alterações morfológicas em útero, tubas e ovários da égua. Belo Horizonte: UFMG, 1991. 253p. Dissertação (Mestrado em Medicina Veterinária) - Escola de Veterinária, 1991.
SILVA FILHO, J.M., SATURNINO, H. M., PALHARES, M. S. et al. Efeito do intervalo entre as duas últimas inseminações e da concentração espermática sobre a fertilidade de éguas inseminadas com sêmen fresco, diluído. In: CONGRESSO BRASILEIRO DE REPRODUÇÃO ANIMAL, 10, 1993, Belo Horizonte. Anais.. Belo Horizonte: CBRA, 1993. p. 264.

TANNUS, R.J., THUN, R. 1995. Influence of endometrial cysts on conception rate of mares. J. Vet. Med., Series A, 42(4):275-283.

VALLE, G.R. Efeito da rufiação e manipulação do sistema genital e transporte de sêmen, em container "Celle"modificado, na avaliação de uma técnica de inseminação artificial em eqüinos. Belo Horizonte: UFMG, Escola de Veterinária. 1997, 349p. Dissertação (Mestrado em Medicina Veterinária), 1997.

VANDERWALL, D.K., WOODS, G.L. Age-related subfertility in the mare. In: ANNUAL CONVENTION OF AMERICAN ASSOCIATION OF EQUINE PRACTITIONERS, 36, 1990, Lexington. Proceedings .... Lexington: American Association of Equine Practitioners, 1991. p.85-89.

VLACHOS, K., PASCHALERI, E. 1965. Research on some factors influencing fertility in solipeds. Bull. Physiol. Path. Reprod. Artif. Insem., 1(1):18-32.

VOSS, J.L., PICKETT, B.W., BACK, D.G. et al. 1975. Effect of rectal palpation on pregnancy rate of nonlactating, normaly cycling mares. J. Anim. Sci., 41(3):829-834.

WAELCHLI, R.O. 1990. Endometrial biopsy in mares under nonuniform breeding management conditions: Prognostic value and relationship with age. Can. Vet. J., 31(5):379-384.

Recebido em: 10/07/98

Aceito em: 11/03/99 